\title{
Research on Sensor Network Construction for Drop and Pull Transportation System
}

\author{
Y.L. Liu \\ Department of Information and Logistics \\ Beijing Vocational College of Finance and Commerce \\ Beijing, China
}

\begin{abstract}
Currently, the drop and pull transportation system is inefficient. In order to solve the problem of low efficiency, this article analysed the current situation of the drop and pull transportation system, and designed a sensor network which is used for drop and pull transportation. This article elaborated the key technology about the sensor network and gave the realized method which is used to build the system architecture. By using the sensor network and the method, we can manage the truck and station more conveniently and more efficiently.
\end{abstract}

Keywords-drop and pull transportation; sensor network; data screening

\section{INTRODUCTION}

Drop and pull transportation is blooming in recent years. Trailer puller is separated from trailer in this model. Puller is driving away directly after connecting with trailer. Transportation is more efficient because puller is running all the time, which means it doesn't need to wait for loading. Currently, domestic road freight transport is high cost because of high empty rate and low operating efficiency. Informatization and intellectualization for drop and pull transportation has become a trend due to the high transport costs and the safety problem etc[1]. This paper will introduce how to identify puller and trailer and how to track cargo automatically, which means improving transparency of transportation system and make it like a network. We want to perceiving and transferring information among station, cargo and trailer. Also I want to integrate the whole sensor network data and connect with logistics public information platform. All the work I have done is aim for solving problems exist in drop and pull transport development, improving drop and pull transport efficiency, improving transparency of public information service platform ,realizing road transport efficient, green and low carbon development.

\section{CURRENT SITUATION ANALYSIS FOR DROP AND PULL TRANSPORT}

Since the 1940s, Europe and the United States and other developed countries began to try drop and pull transportation. After years of development, drop and pull transport in western developed countries and industrialized countries has made great progress and become the main transportation. Drop and pull transportation accounted for $70 \% \sim 80 \%$ of road freight transportation. The ratio of puller and trailer reached a 1:2. 5 ; It has been widely used in emerging industrialized countries such as Singapore, South Korea, the ratio of the puller and trailer of Singapore reached 1:7. Drop and pull transportation is an advanced mode because it is nice for environment and economic. According to authority estimate, drop and pull transport improve vehicle transport efficiency by 30\% 50\%, reduce cost by $30 \% \sim 40 \%$, and reduce oil consumption by $20 \% \sim 30 \%$.

China is restrict by many factors, Drop and pull transportation is still in its infancy because of many restricted factors. Puller and trailer is about 1:1.9, drop and pull transportation road freight total turnover is less than $10 \%$ of the road transportation. I summarize the restricted factors as: (1) Drop and pull transport information is not accurate because of lack of information collection equipment and low sensor network coverage, we can't the effectively monitored truck location, trailer, cargos, stations, and road. (2) Data docking is difficult because each drop and pull transport system standard are not identical and the underlying sensor data varies from each other. It can't support logistics information platform support with only location data providing. (3) Each system works alone and don't connect with each other. We need a platform to realize data exchange among each system in order to improve logistic efficiency[2]. In order to solving these problem, the system designed in this paper will use RFID, long-distance communication and sensor technology to build IOT system for truck, station and trailer. A systematic and reasonable system for drop and pull transportation sensor networks is built to improve logistic efficiency.

\section{RESEARCH ON DROP AND PULL TRANSPORTATION SENSOR NETWORKS}

Whole drop and pull transportation system is divided into three parts: trucks, stations and carriages. Trucks stand for puller, stations stand for parking place, and carriages stand for cargo and trailer. The three parts is closed linked. System architecture is shown in figure I .

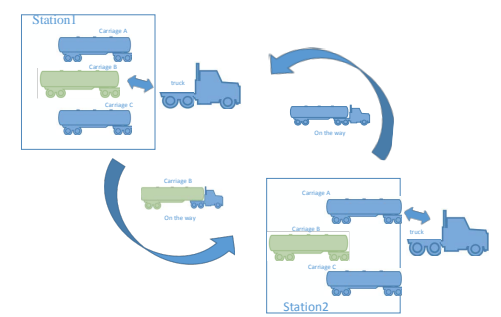

FIGURE I .DROP AND PULL TRANSPORT SYSTEM ARCHITECTURE.

As figure I show, puller in station 1 locked with carriage B quickly, then it moved to station 2.After unloading 
carriage B, puller locked with carriage A and went back to station 1 . We can get a conclusion that accurate recognition of truck Icarriagelstation in whole system and monitoring trucks and carriages in real-time is two crucial factors to realize system transparent, it is also important for building logistics sensor network for drop and pull transportation[3].

\section{A. Research on Drop and Pull Transportation Truck Sensing System}

Drop and pull transport truck sensing system is composed of GPS/BEIDOU positioning device, RFID identification device, communications device and biological recognition system. Drop and pull transportation truck sensing system architecture is shown in figure II.

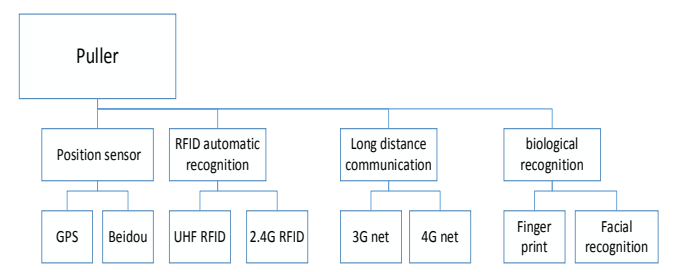

FIGURE II.TRUCK SENSOR SYSTEM ARCHITECTURE.

GPS/BEIDOU positioning device is used for positioning the puller position, obtaining truck location data. RFID identification device is used for identify whether puller is locked with correct carriages. We use UHF RFID or $2.4 \mathrm{G}$ RFID technology to identify puller and carriage because this kind of RFID features long distance recognition in outdoor. We put RFID identification equipment on puller, which is used to identify RFID tag on carriage. In this w ay we can avoid problems such as puller locked with wrong carriage or puller drive away without carry anything. Communication device is used for driver contacting with platform and data transmission between puller and system. $3 \mathrm{~g} / 4 \mathrm{~g}$ technology is suitable for long distance transport. By this way we can achieve high coverage of road network communications and effective bandwidth of data sending \receiving. Biological recognition system is used to identify driver before he leaving station, confirm the legality of driver, and achieve lean management. This system can be fingerprint identification or facial recognition, which can accurately give driver's personal information. Driver's physiological information can also be given.

\section{B. Research on Drop and Pull Transportation Station System}

Station sensing system is used for managing truck in the station, such as truck position and whether carriage is empty. Station system confirms truck position and empty carriage and dispatch forklift or packing workers to load cargo. Workers identify the empty carriage by hand-held terminals (PDA) and start loading. After finish the loading work, workers sent loading information to system via handheld PDA. Station sensing system is composed of RFID positioningไrecognizing device and handheld identification terminal PDA .System architecture is shown in figure III. Station RFID positioning Irecognizing device is a kind of RTLS system, which have the RFID positioning function. It can be used for positioning puller and carriage position for real-time. Handheld identification terminal PDA is used for identify puller, carriage, cargo and position information. It shall be equipped with the GPS data acquisition function and RFID data read Iwrite function.

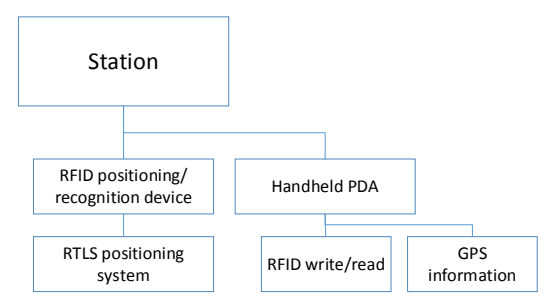

FIGURE III.STATION SENSOR SYSTEM ARCHITECTURE.

\section{Carriage System Research}

Carriage system is composed of GPS unit, UHF RFID tags, multi-purpose sensing device; system architecture is shown in figure IV.Multi-purpose sensing device including tire pressure monitor, temperature sensors, humidity sensors, radiation level sensors, light sensors, tilt sensor (selected according to the actual need).Multi-purpose sensing device is used to collect information about inside carriage. UHF RFID tag is used to identify carriage. Sensor in carriage is used to monitor cargo in carriage. Due to cargo maybe agricultural product, industrial chemicals or something else, we need to choose different types of sensors to detect different cargo.

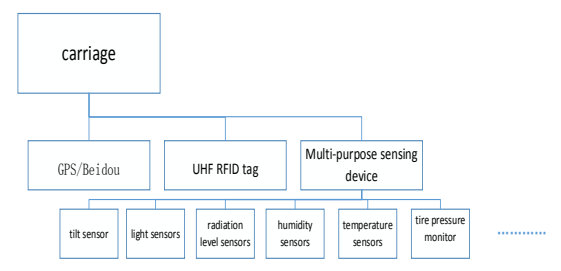

FIGURE IV. CARRIAGE SYSTEM.

\section{Sensor Middleware Research}

Cloud terminal middleware technology is critical for logistics information service system to identify the underlying sensor networks. Only in this way can we make all kinds of public service platform connect with drop and pull transport sensor network. Middleware technology solves the problem of device heterogeneity and invalid data interference. Preliminary data analysis and judgment is made as well. Due to the system adopts cloud model for middleware, the key to realize this system include two parts: the first part is hardware support, the second part is the data analysis and screening.

1) Middleware support hardware technology: There are many types of devices among cloud terminal. Each device has a lot of protocol types. For example, RFID used in drop and pull transport sensor network has different protocols. How to identify these devices and make effective control is one of the critical problems to solve in this paper. Different protocols have different data packet type, which contained baud rate, data bits, parity, and stop bits. Command data packet format is shown in table I, Response data packet format is shown in table II. 
TABLE I. COMMAND DATA PACKET FORMAT.

\begin{tabular}{|l|l|l|l|}
\hline Data segment & SOF & LENGTH & CMD \\
\hline length & 1 & 1 & 1 \\
\hline \multicolumn{4}{|l|}{} \\
\hline Data segment & PAYLOAD & CRC-16 & EOF \\
\hline length & $<254$ & 2 & 1 \\
\hline
\end{tabular}

TABLE II. RESPONSE DATA PACKET FORMAT

\begin{tabular}{|l|l|l|l|l|}
\hline $\begin{array}{l}\text { Data } \\
\text { segment }\end{array}$ & SOF & LENGTH & CMD & STATUS \\
\hline length & 1 & 1 & 1 & 1 \\
\hline \multicolumn{5}{|l|}{} \\
\hline $\begin{array}{l}\text { Data } \\
\text { segment }\end{array}$ & PAYLOAD & CRC-16 & EOF & \\
\hline length & $<254$ & 2 & 1 & \\
\hline
\end{tabular}

Here we make an example to show UHF 18000-6 c protocol identification: when a user connected to RFID read-write device and need recognition, the first thing he need do is create two data packet format table in program. Then he sends the data in different structure and different format. Data is need to be transcoded when received, then data is filled in response packet format table and wait for being parsed.

2) Data screening and event analyzing technology: Basic principle of data analyzing and screening is: semantic rules in middleware is used for confirming device events, a preliminary analysis is done according to the configuration of semantic rule base. If data is needed to be screened, it won't be sending out. If data is important information, system will prompt user to pay attention for it. Mature event description language can be used in the program is not much, the CDL description language (Complex - the Event Description Language) is one of the languages which has been widely used. This language is like mathematical expression, which can be used to describe, match, screen and converse event accurately.

\section{CONCLUSION}

This paper put forward the overall drop and pull logistics transportation sensor network system architecture after analyzing drop and pull transportation sensor networks model. Middleware technology was discussed to serve for public service platform in order to improve platform working efficiency because various data is provided. The study has a certain reference and guidance significance for drop and pulls sensor networks deploying.

\section{REFERENCE}

[1] Xin Manyu. Truck schedule research based on drop and pull transport net model [J]. Logistics science,2014,1:254-258.

[2] Gao Hui. Intelligent schedule system for drop and pull transportation [J]. Satellite and Internet ,2013,3:90-92.

[3] Hou Baohua. Intelligent file management based on RFID [J].Haerbin commerce university joural,2014,2:249-252,256. 